\title{
Practifde $\mathfrak{A} \mathfrak{n} \mathfrak{l} \mathfrak{e}$ it $\mathfrak{u} \mathfrak{n} \mathfrak{g}$ \\ $\mathfrak{z} \mathfrak{u} \mathfrak{r} \mathfrak{F} \mathfrak{u} \mathfrak{h} \mathfrak{x} \mathfrak{u} \mathfrak{n} \mathfrak{g}$
}

\section{b e $x$

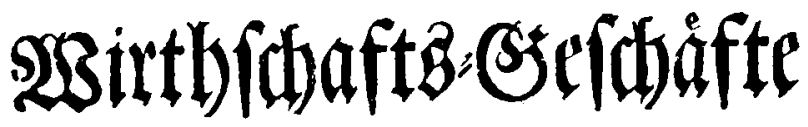

fin

\section{angehende Landwirtbe,}

von

Dr. Frieorid Sar! Suftab Gericfe, Dber = Lumtmann uno mitgliebe ocr Lanowirtbjhaftb = Be: fellidaft zu Getle unb Eeipzig 26 .

Dritter ateit.

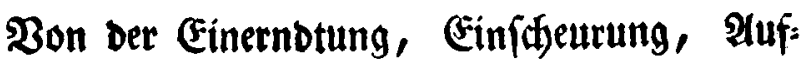
bewahrung und Berfilberung der gewonnenen FBiefen, und Feld = (Erzeugntfe; aud) practif uno Effígbrauerei.

3weite vermelyte unb verbefferte Iruflage.

Mit 8 Rupfetafel

Berlin I 8 I 5.

3n ber Realfaulbudbandang. 

$\mathfrak{D} \mathfrak{e} \mathfrak{m}$

\section{Serte Gottlob গatbufius \\ ber}

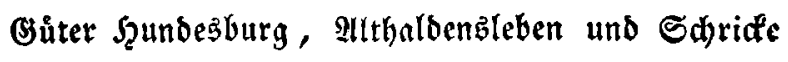
Erv = unb Berid)täherrn, wo is met biefen Theil feiner Suriften ค แ เ nadbartidber Siebe und Freund(áaft Der $\mathfrak{B e r f a f f e r}$. 

3uneignungefwriftent laben gemeiniglidf auf Den $\mathfrak{B e r f a f f e r}$ Den $\mathfrak{A r g w o b n}$, als wolle er mit Shrotection ober einem sefchenpe bes lofnnt fern, und mandfen mag diefer Irg= wofn audf mit Teefft treffen. Darum liebe idf bie Sadbe nidft.

If) bitt freilich eirmahl burdf einent verefrten $\mathfrak{M a n n}$, ben werfforbenten $\mathfrak{B}$. $\mathfrak{P r}$. $\mathfrak{H}$. in $\mathfrak{S}$. berwogen worden, eine Zueigs nungäfdrift, weldhe aus biejes hod headt)= teten Mannes Feder Foß́, unter meinent Namen einem Bande meines $\mathfrak{B e r t a ̉ ~}$ vors bructen za laffen; aber es that mir nacty= ber leid, weil idf fajefe Untbeile barúber hơren muste.

$\mathfrak{2 B e n n}$ inder ein Freund, feinem $\mathfrak{R a d f s}$ 
bar und Freunbe, aus inniger Pdftung und Buweigung ein B̉udf zueignet, wean $D a=$ bei Das ólonomirace Publifum geninnt, indem es einen fich lim bie Eandwitthid)aft fo vertient madenben Mann náfer len: nen lernt; fo lann ber Berfaffer leines Eigennuteces berfulldigt werden.

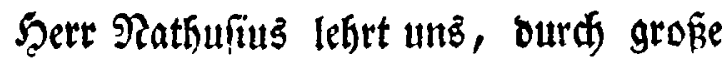
Opfer, mit einer beifptellofen Thátig= leit uno einem nadababungsmuroigen Fleibe, unfere gewonnenen Wirthifhaftb= probulte auf bie mannigfadjte veredlen und su einem hoberen Greldwerthe uminfdaffert. Er lefint uns, ausz den Runt: Eelrúben, die unfer $\mathfrak{B}_{0}$ onn producirt, eis nen Dem indifuien vollfominen gleicfen Sucler mit anjefnlidein Gewinn fa ma: 
đen, aus bem gewonnenen SBeigen und bet Gerffe, ein bem englifhen Biere gleidjen= Des Getrån 'u bereiten, aus andern $\mathfrak{G e}=$ treide und Sartoffeln, Dura neue $\mathfrak{B o r}=$ Eefrungen, eine grópere Quantitåt B̉ranote: wein zu getwinnen, aus biefem wieber allerlei 2quavite, Eiqueure und Fiecf):

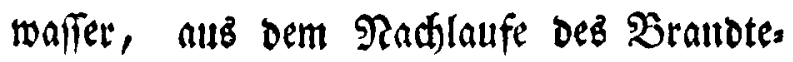
weins uno fonftiger 2 fbgånge aber man: d)erlei Effig = 2rten ju ergeugen, Den aus Dem Reps gewonnenen Dele, ourd dos Faffiniren, im Brennen Dem Baumóle

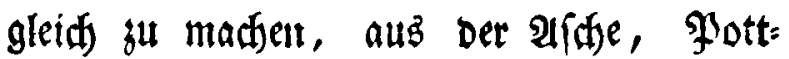

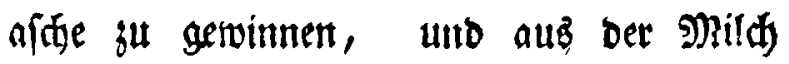

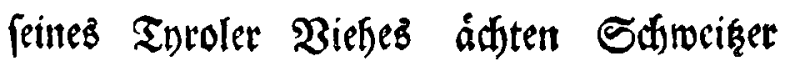
Fåfe zu probuciren :c.; burfh bie $\mathfrak{A b}=$ falle aber ben Ertrag deż 2tckerż zu er= biffen. 
Grit wim fursen auf bem einen Gute lauter Toroler, auf bem andern Friefindes, uno auf Dem britten Jựtlánoer Sornvié) auffallen, und vergleidsende $\mathfrak{B e r f u c h e ~ u ́ b e r ~}$ Den Ertrag anftellen.

5Benn mun $\mathfrak{G e r r}$ Ratfufus in allent diefen Miffemfitaften, auf bie uneigens

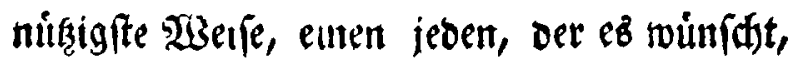
unterridtet und aus feinen gemachten Er $_{\mathrm{r}}$ fafirungen Eein Gichermnizi madts; fo bers bient cine dpontumifids: Shrift wobl, daß fie fein $\mathfrak{R a m e}$ jiere, uno barum ffefe er bier. 